\title{
Antileishmanial activity of diterpene acids in copaiba oil
}

\author{
Adriana Oliveira dos Santos' ${ }^{1}$, Erika Izumi' ${ }^{1}$ Tânia Ueda-Nakamura² ${ }^{2}$ Benedito Prado Dias-Filho ${ }^{1,2}$, \\ Valdir Florêncio da Veiga-Júnior ${ }^{3}$, Celso Vataru Nakamura ${ }^{1,2} /+$ \\ 1Programa de Pós-Graduação em Microbiologia, Universidade Estadual de Londrina, Londrina, PR, Brasil ²Programa de Pós-Graduação \\ em Ciências Farmacêuticas, Universidade Estadual de Maringá, Maringá, PR, Brasil Departamento de Química, \\ Instituto de Ciências Exatas, Universidade Federal do Amazonas, Manaus, AM, Brasil
}

Leishmaniasis is a neglected tropical disease. According to the World Health Organization, there are approximately 1.5-two million new cases of cutaneous leishmaniasis each year worldwide. Chemotherapy against leishmaniasis is based on pentavalent antimonials, which were developed more than a century ago. The goals of this study were to investigate the antileishmanial activity of diterpene acids in copaiba oil, as well as some possible targets of their action against Leishmania amazonensis. Methyl copalate and agathic, hydroxycopalic, kaurenoic, pinifolic and polyaltic acids isolated from Copaifera officinales oleoresins were utilised. Ultrastructural changes and the specific organelle targets of diterpenes were investigated with electron microscopy and flow cytometry, respectively. All compounds had some level of activity against $\mathrm{L}$. amazonensis. Hydroxycopalic acid and methyl copalate demonstrated the most activity against promastigotes and had 50\% inhibitory concentration $\left(I C_{50}\right)$ values of 2.5 and $6.0 \mu \mathrm{g} /$ $m L$, respectively. However, pinifolic and kaurenoic acid demonstrated the most activity against axenic amastigote and had $I C_{50}$ values of 3.5 and $4.0 \mu \mathrm{g} / \mathrm{mL}$, respectively. Agathic, kaurenoic and pinifolic acid caused significant increases in plasma membrane permeability and mitochondrial membrane depolarisation of the protozoan. In conclusion, copaiba oil and its diterpene acids should be explored for the development of new antileishmanial drugs.

Key words: Leishmania amazonensis - leishmaniasis - Copaifera - diterpene acids

Leishmania is a genus of protozoan parasites that are transmitted by the bite of the sandfly and cause diseases collectively known as leishmaniasis (Kaye \& Scott 2011). The disease has varying clinical manifestations that range from self-healing cutaneous and mucocutaneous skin ulcers to a fatal visceral form of the disease called visceral leishmaniasis or kala-azar (Murray et al. 2005, Murray 2010, Aït-Oudhia et al. 2011). Leishmaniasis is one of the most significant of the neglected tropical diseases (Kaye \& Scott 2011). According to the World Health Organization (WHO), there are approximately 1.5-two million new cases of cutaneous leishmaniasis each year worldwide. This disease is endemic in 88 countries, with a total of 350 million people at risk. It is believed that 12 million people are affected by leishmaniasis worldwide (WHO 2011). Despite the overwhelming impact of these parasites, there are still many aspects of the mechanisms of pathogenesis and how these organisms survive in the host that remain to be elucidated.

There are no vaccines available for leishmaniasis and current treatments with antimonials suffer from several limitations (Gelb \& Hol 2002, Cruz et al. 2009). The main alternative drugs include pentamidine, amphotericin $\mathrm{B}$ and amphotericin B encapsulated in liposomes. This en-

Financial support: CNPq, CAPES, FINEP, PRONEX/Fundação

Araucária, UEM/COMCAP

+Corresponding author: cvnakamura@uem.br

Received 2 April 2012

Accepted 5 September 2012 capsulation reduces the occurrence of side effects; however, relapses still occur and the drug remains extremely expensive. Miltefosine, an alkylphospholipid, was developed as an oral antineoplastic agent and has become the first oral treatment for leishmaniasis in some countries (Seifert 2011, Tiuman et al. 2011). Natural products are a primary source of many pharmaceuticals used today. Several studies have shown that the use of copaiba oil positively demonstrated antileishmanial activity. Previously, our group observed that copaiba oils obtained from different species of Copaifera show activity against promastigote forms of Leishmania amazonensis (Santos et al. 2008). Significant antileishmanial activity of copaiba oil from Copaifera reticulata was demonstrated against axenic amastigote and intracellular amastigote forms of the parasite. Additionally, we demonstrated that copaiba oil oral treatment caused a significant reduction in the average lesion size in mice (Santos et al. 2011). More recently, Santos et al. (2012) used electron microscopy to demonstrate the morphological and ultrastructural changes in L. amazonensis treated with copaiba oil from $C$. reticulata and used flow cytometry to investigate specific organelles as targets for copaiba oil. Therefore, this study investigated the antileishmanial activity of diterpene acids from copaiba oil, as well as some possible targets of their action against the promastigote and axenic amastigote forms of $L$. amazonensis.

\section{SUBJECTS, MATERIALS AND METHODS}

Compounds - The compounds utilised in this study include agathic acid [labd-8(20)-13-dien-15,19-dioic acid], hydroxycopalic acid [3ß-hydroxylabda-8(20),13-dien-15oic acid], kaurenoic acid [kaur-16-ene-18-oic acid], methyl copalate [methyl labd-8(20),13-dien-15-oate], pinifolic 
acid [labd-8(20)-ene-15,18-dioic acid] and polyaltic acid [15,16-epoxylabda-8(20),13(16),14-trien-19-oic acid]. All compounds were isolated from Copaifera oleoresins $(\geq$ $90 \%$ by nuclear magnetic resonance) using potassium hydroxide-impregnated silica gel chromatography according to the procedures used by Izumi et al. (2012). Each compound was first dissolved in dimethylsulfoxide and then added to the appropriate medium.

Parasites - Promastigote forms of L. amazonensis (WHOM/BR/75/Josefa), which were originally isolated by Cesar Augusto Cuba-Cuba (Universidade de Brasília, Brazil) from a human case of diffuse cutaneous leishmaniasis, were cultured at $25^{\circ} \mathrm{C}$ in Warren's medium (brain-heart infusion plus haemin and folic acid) supplemented with $10 \%$ heat-inactivated foetal bovine serum (FBS) (Gibco Invitrogen Corporation, New York, USA) in a tissue flask. Axenic amastigote cultures obtained by in vitro transformations of infective promastigotes (Ueda-Nakamura et al. 2001) were incubated at $32^{\circ} \mathrm{C}$ in Schneider's insect medium (Sigma Chemical Co, St. Louis, Missouri, USA), pH 4.6, with 20\% FBS.

Antileishmanial activity - L. amazonensis promastigotes $\left(1 \times 10^{6}\right.$ parasites $\left./ \mathrm{mL}\right)$ were inoculated in a 24-well plate containing Warren's medium supplemented with $10 \%$ inactivated FBS with different concentrations of diterpene acids and they were incubated at $25^{\circ} \mathrm{C}$ for 72 $\mathrm{h}$. The cell density associated with each concentration was determined by counting cells with a haemocytometer (Improved Double Neubauer). The experiments with amastigote forms were performed as described above using Schneider's Insect Medium, $\mathrm{pH}$ 4.6, supplemented with $20 \%$ inactivated FBS and incubated at $32^{\circ} \mathrm{C}$ for 72 h. Amphotericin B was used as a positive control. Controls containing $0.5 \%$ dimethyl sulfoxide (Sigma Chemical Co, St. Louis, MO, USA) and medium alone were also included. The $50 \%$ inhibitory concentration $\left(\mathrm{IC}_{50}\right)$ was determined by logarithm regression analysis of the data obtained (Santos et al. 2012).

Cytotoxic activity - Human blood was collected from healthy donors and the erythrocytes were used for cytotoxic evaluation of the diterpene acids. Prior to the experiment, the blood was defibrinated and washed in glycosylated saline. Erythrocytes were inoculated in 96-well plates, in 3\% glycosylated saline, with different concentrations of the diterpene acids, followed by incubation for $3 \mathrm{~h}$ at $37^{\circ} \mathrm{C}$. The absorbance of the supernatant at $550 \mathrm{~nm}$ was measured and compared with that of wells treated with $1 \%$ Triton X-100 to calculate the percentage of haemolysis (Izumi et al. 2012).

Ultrastructural analysis - Promastigote forms $(1 \mathrm{x}$ $10^{6}$ parasites $/ \mathrm{mL}$ ) were treated with hydroxycopalic acid at $2.5 \mu \mathrm{g} / \mathrm{mL}$ for $72 \mathrm{~h}$ and then processed for electron microscopy. Parasite cells were harvested, washed twice with PBS and fixed with $2.5 \%$ glutaraldehyde in $0.1 \mathrm{M}$ sodium cacodylate buffer at $4^{\circ} \mathrm{C}$. For scanning electron microscopy (SEM), promastigote forms were fixed as described previously. Thereafter, small drops of the sample were placed on a specimen support with polyL-lysine. Subsequently, the samples were dehydrated in graded ethanol, critical-point-dried in $\mathrm{CO}_{2}$, coated with gold and observed using a Shimadzu SS-550 SEM. For transmission electron microscopy (TEM), cells were post-fixed in a solution containing $1 \% \mathrm{OsO}_{4}, 0.8 \%$ potassium ferrocyanide and $10 \mathrm{mM} \mathrm{CaCl}_{2}$ in $0.1 \mathrm{M}$ cacodylate buffer, dehydrated in an increasing acetone gradient and embedded in Epon ${ }^{\circledR}$ resin. Next, ultrathin sections were stained with uranyl acetate and lead citrate and images were obtained on a Zeiss 900 TEM (Santos et al. 2012).

Determination of the mitochondrial membrane potential - Axenic amastigote forms of L. amazonensis (5 x $10^{6}$ cells $/ \mathrm{mL}$ ), either untreated or treated with $100 \mu \mathrm{g} /$ $\mathrm{mL}$ of diterpene acids at $32^{\circ} \mathrm{C}$ for $3 \mathrm{~h}$, were washed and incubated with $5 \mathrm{mg} / \mathrm{mL}$ of rhodamine 123 (Rh123) for $15 \mathrm{~min}$ at $37^{\circ} \mathrm{C}$ to evaluate the mitochondrial membrane potential. The compound carbonyl cyanide m-chlorophenylhydrazone (CCCP) was used as a positive control. All of the material was kept on ice until analysis. Data acquisition and analysis were performed with a FACSCalibur flow cytometer (Becton Dickinson, Rutherford, NJ, USA) equipped with the CellQuest software (Joseph Trotter, Scripps Research Institute, La Jolla, CA, USA). A total of 10,000 events were acquired in the region previously established as that corresponding to the parasites. Alterations in the fluorescence of Rh123 were quantified as the per cent of reduction of the fluorescence compared with the control (untreated parasites) (Santos et al. 2012).

Cell viability - Cell viability was assessed by staining the cells with propidium iodide (PI). Axenic amastigotes $\left(5 \times 10^{6}\right.$ cells $\left./ \mathrm{mL}\right)$, either untreated or treated with $100 \mu \mathrm{g} / \mathrm{mL}$ of diterpene acids at $32^{\circ} \mathrm{C}$ for $3 \mathrm{~h}$, were harvested and washed with PBS buffer. Then, the parasite cells were stained with PI $(20 \mu \mathrm{g} / \mathrm{mL}$ for 5 min) according to the manufacturer's instructions. Amphotericin B was used as a positive control. The mean fluorescence intensity was analysed using FACSCalibur and CellQuest software. A total of 10,000 events were acquired in the region established as that corresponding to the parasites (Santos et al. 2012).

Statistical analysis - One-way ANOVA and Tukey's test were performed with GraphPad Prism 4 (GraphPad Software, San Diego, California, USA). Values of $p<$ 0.05 were considered significant.

\section{RESULTS}

The compounds tested were agathic acid, hydroxycopalic acid, kaurenoic acid, methyl copalate, pinifolic acid and polyaltic acid, which were isolated from Copaifera. Hydroxycopalic acid and methyl copalate demonstrated the most activity, with $\mathrm{IC}_{50}$ values of 2.5 and $6.0 \mu \mathrm{g} / \mathrm{mL}$, respectively, against promastigote forms of $L$. amazonensis (Table). Amphotericin $\mathrm{B}$ had an $\mathrm{IC}_{50}$ of $0.06 \mu \mathrm{g} /$ $\mathrm{mL}$ against promastigotes after $72 \mathrm{~h}$ of treatment. The ANOVA $(p<0.05)$ indicated significant differences between the isolated compounds and the control group.

Diterpene acids had low toxicity to mammalian erythrocytes. Pinifolic and polyaltic acids did not cause lysis except at the maximum concentration tested. The 
haemolysis rates of methyl copalate $(500 \mu \mathrm{g} / \mathrm{mL})$ and agathic acid $(350 \mu \mathrm{g} / \mathrm{mL})$ were both $50 \%$. Kaurenoic and hydroxycopalic acids were more toxic (Table).

The morphological and ultrastructural changes of the parasites were investigated after treatment with hydroxycopalic acid, as shown in Fig. 1A-D. Using SEM, it was possible to observe the typical elongated shape of untreated cells (Fig. 1A). However, when the parasites were treated with hydroxycopalic acid, we observed notable morphological changes, such as the appearance of rounded cells (Fig. 1D), rupture of the plasma membrane with loss of the cell contents (Fig. 1B) and significant alterations of the flagellar membrane (Fig. 1C). TEM results are shown in Fig. 1E-H. Untreated promastigotes showed no plasma membrane alterations and contained organelles with normal morphology (Fig. 1E). In parasites treated with hydroxycopalic acid, the most prominent effect was swollen mitochondria with the appearance of concentric membrane structures inside the organelle (Fig. 1F-H). Other ultrastructural alterations induced by hydroxycopalic acid were abnormal chromatin condensation and intense exocytic activity in the region of the flagellar pocket (Fig. 1G).

Diterpene acids were also evaluated with regard to their actions against axenic amastigote forms of $L$. amazonensis. The most active acids in inhibiting the proliferation of axenic amastigotes were pinifolic acid and kaurenoic acid, with $\mathrm{IC}_{50}$ values of 4.0 and $3.5 \mu \mathrm{g} /$ $\mathrm{mL}$, respectively (Table). Amphotericin $\mathrm{B}$ had an $\mathrm{IC}_{50}$ of $0.23 \mu \mathrm{g} / \mathrm{mL}$ against amastigote forms. The ANOVA $(p<0.05)$ showed significant differences between the isolated compounds and the control group.

As previously described (Santos et al. 2012), PI labelling was employed to evaluate the cell viability of the parasite. PI is a well-known DNA marker; it is a fluorescent dye that binds specifically to DNA. As shown in Fig. 2, the relative numbers of PI-positive events were measured and compared with the upper left quadrant gated percentage of positive controls (75.9\%) (Fig. 2H) and negative controls (11.4\%) (Fig. 2G). Cell membrane integrity was affected mainly by agathic acid $(63.4 \%)$, kaurenoic acid (60.8\%) and pinifolic acid (68.4\%) (Fig. $2 \mathrm{~A}, \mathrm{C}, \mathrm{E})$. These diterpene acids induced a considerable increase in plasma membrane permeability in the axenic amastigote forms of $L$. amazonensis. The percentages of gated cells treated with hydroxycopalic acid, methyl copalate and polyaltic acid (Fig. 2B, D, F) were similar to those in the negative control (Fig. 2G). All treatments were applied for $3 \mathrm{~h}$ at $32^{\circ} \mathrm{C}$.

Flow cytometry with Rh123 was used for membrane potential measurements. The results showed a marked decrease in the upper right gated population percentage after treatments with agathic acid $(27.5 \%)$, kaurenoic acid $(24.4 \%)$ and pinifolic acid (32.4\%) (Fig. 2A, C, E), indicating depolarisation of the mitochondrial membrane potential in the cells after treatment with these acids at $100 \mu \mathrm{g} / \mathrm{mL}$ for $3 \mathrm{~h}$ at $32^{\circ} \mathrm{C}$. Similarly, a decrease in membrane potential was also observed following treatment with the standard drug CCCP (53.1\%) (Fig. 2H) at 200 $\mu \mathrm{M}$ for $3 \mathrm{~h}$ at $32^{\circ} \mathrm{C}$. In contrast, the membrane potential was maintained in untreated cells (93.7\%) (Fig. 2G).

\section{DISCUSSION}

In this study, we tested the in vitro antileishmanial activity of isolated compounds from copaiba oil against the promastigote and axenic amastigote forms of L. amazonensis and obtained promising results. Recently, Izumi et al. (2012) evaluated diterpenes from Copaifera with trypanosomal activity and showed that they were more active against intracellular forms of $L$. amazonensis. Similarly, in our study, the majority of the compounds were more effective against amastigotes than promas-

TABLE

Antileishmanial and cytotoxic activity of diterpenes from copaiba oil

\begin{tabular}{|c|c|c|c|c|}
\hline \multirow[b]{3}{*}{ Compounds } & \multicolumn{4}{|c|}{$\mu \mathrm{g} / \mathrm{mL}$} \\
\hline & \multicolumn{2}{|c|}{$\mathrm{IC}_{50}$} & \multirow{2}{*}{$\frac{\mathrm{CC}_{50}}{\text { Erythrocytes }}$} & \multirow{2}{*}{$\frac{\mathrm{IS}}{\mathrm{CC}_{50} / \mathrm{IC}_{50} \mathrm{ama}}$} \\
\hline & Promastigote & Amastigote & & \\
\hline Agathic acid & $28.0 \pm 1.5^{b}$ & $17.0 \pm 2.0^{b}$ & $350.0 \pm 3.4$ & 20.6 \\
\hline Hydroxycopalic acid & $2.5 \pm 0.4^{a}$ & $18.0 \pm 1.5^{b}$ & $40.0 \pm 2.4$ & 2.2 \\
\hline Kaurenoic acid & $28.0 \pm 0.7^{b}$ & $3.5 \pm 0.5^{a}$ & $140.0 \pm 17.0$ & 40.0 \\
\hline Methyl copalate & $6.0 \pm 0.9^{a}$ & $14.0 \pm 1.0^{b}$ & $500.0 \pm 3.0$ & 35.7 \\
\hline Pinifolic acid & $70.0 \pm 8.0^{b}$ & $4.0 \pm 0.4^{a}$ & $>500.0$ & $>125.0$ \\
\hline Polyaltic acid & $35.0 \pm 2.0^{b}$ & $15.0 \pm 1.0^{b}$ & $>500.0$ & $>33.3$ \\
\hline Amphotericin B & $0.06 \pm 0.0$ & $0.23 \pm 0.0$ & ND & ND \\
\hline
\end{tabular}

$a$ : significant difference when compared to others diterpenes acids (ANOVA test; $\mathrm{p}<0.05$ ); $b$ : no significant difference when compared to hidroxycopalic acid and methyl copalate (promastigote test), kaurenoic acid and pinifolic acid (amastigote test) (ANOVA test; $\mathrm{p}<0.05)$. Significant differences between compound's activity and control cell growth, ANOVA $(\mathrm{p}<0.05)$. Values represent the mean \pm standard deviation of at least three experiments performed in duplicate. $\mathrm{CC}_{50}$ : cytotoxic concentration of $50 \%$; $\mathrm{IC}_{50}$ : inhibitory concentration of 50\%; ND: not determined; SI: selectivity index between erythrocytes and amastigote forms. 

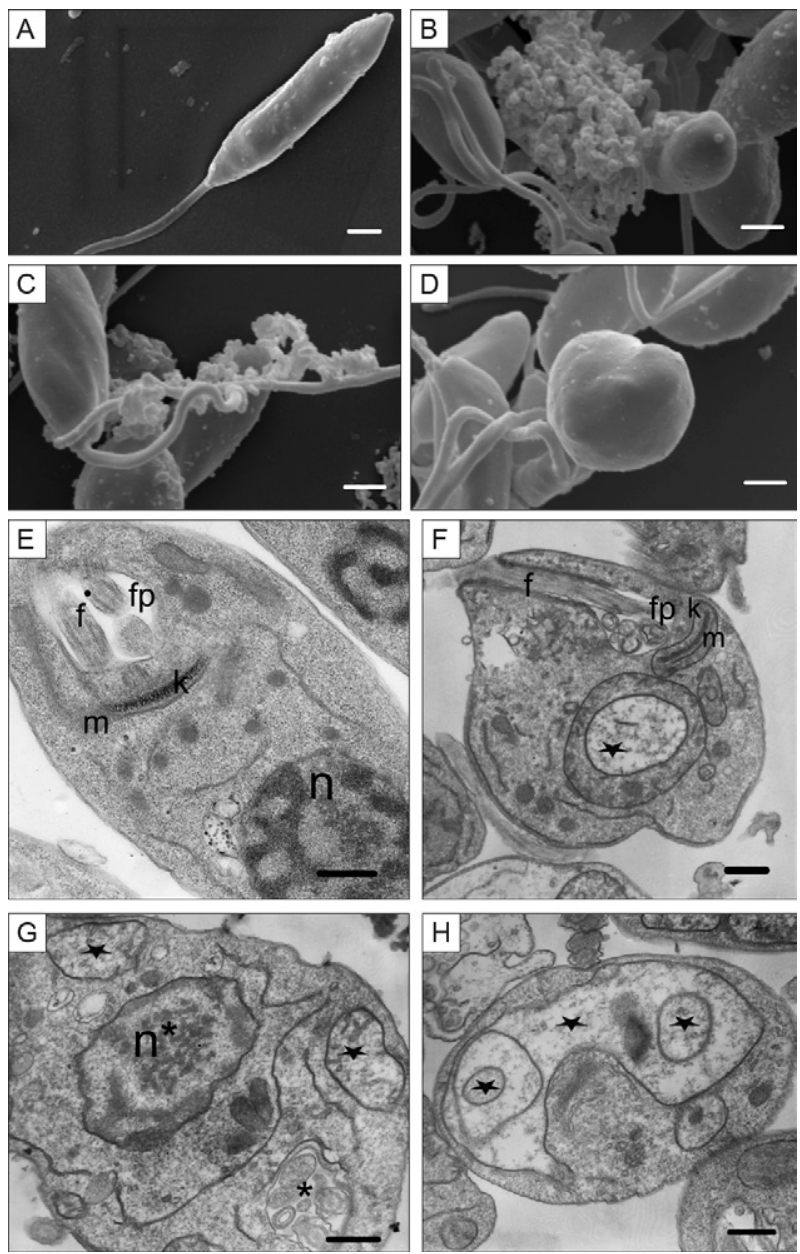

Fig. 1: scanning (SEM) and transmission (TEM) electron micrographs of promastigote forms of Leishmania amazonensis treated with 2.5 $\mu \mathrm{g} / \mathrm{mL}$ hydroxycopalic acid for $72 \mathrm{~h}$. A: control (SEM); B-D: treated parasites; E: control (TEM); F, G: treated parasites. The hydroxycopalic acid led to changes in the mitochondria (stars), exocytic activity in the region of the flagellar pocket (asterisk) and abnormal chromatin condensation nuclear alterations (n*). n: nucleus; f: flagellum; fp: flagellar pocket; k: kinetoplast; m: mitochondrion. Bars $=1 \mu \mathrm{m}$.

tigotes, except for methyl copalate and hydroxycopalic acid, which exhibited greater inhibition against promastigotes; specifically, these compounds demonstrated an inhibition of $50 \%$ against intracellular trypanosomes at 0.8 and $0.6 \mu \mathrm{g} / \mathrm{mL}$, respectively (Izumi et al. 2012). Additionally, Santos et al. (2008) reported activity of copaiba oil from $C$. reticulata against intracellular amastigotes, with an $\mathrm{IC}_{50}$ value of $20.0 \mu \mathrm{g} / \mathrm{mL}$.

The ranges of activity of some diterpenes vary considerably depending on the evolutive form of the parasite. Pinifolic acid presented an $\mathrm{IC}_{50}$ of $70 \mu \mathrm{g} / \mathrm{mL}$ for promastigotes and only $4 \mu \mathrm{g} / \mathrm{mL}$ for amastigotes, which is almost an 18-fold reduction in the concentration of the drug required for the same percentage of inhibition. The IC of kaurenoic acid varies from $3.5-28 \mu \mathrm{g} / \mathrm{mL}$, which is also a very significant variation in the amount of drug needed.
Almost all diterpenes exhibited low toxicity to mammalian red blood cells, which is an important finding because the medication used to treat leishmaniasis needs to be absorbed by blood cells and distributed by these cells through the circulatory system to infected regions of the body.

In accordance with the toxicity results, the selectivity index (SI) of methyl copalate was determined to be almost 84 . For pinifolic and polyaltic acids, it was not possible to calculate the SI because no toxicity was observed, except at the maximum concentration tested. Hydroxycopalic acid had a SI of 16, while agathic acid was 12 times more toxic to the parasite than to the host cells. The SI of kaurenoic acid, which was the least selective of the compounds tested, was 5. Methyl copalate exhibited the best selectivity against trypanosomes, in accordance with the results of Izumi et al. (2012).

Others studies with copaiba oils have often demonstrated morphological changes and alterations in the mitochondrial ultrastructure. Santos et al. (2012) showed that $C$. reticulata treatment led to rupture of the plasma membrane with loss of its contents in amastigote forms of L. amazonensis. Additionally, Copaifera martii has been demonstrated to cause the appearance of aberrantshaped cells (Santos et al. 2011). In both Copaifera species, the most notable ultrastructural effect of the treatment was swollen mitochondria. Several studies have demonstrated changes in the mitochondria of Leishmania species treated with several antileishmanial agents (Delorenzi et al. 2001, Rosa et al. 2003, Santa-Rita et al. 2004, Ueda-Nakamura et al. 2006, Rodrigues et al. 2007, Santos et al. 2010). These studies reported that significant alterations in the mitochondria led to the loss of cell viability and confirmed the importance of this organelle for the parasite's viability.

Izumi et al. (2012) evaluated ultrastructural changes in $T$. cruzi and observed plasmatic and mitochondrial membrane alterations after treatment with terpenes from Copaifera. In epimastigotes, the extracellular replicative form, hydroxycopalic acid caused organelle disorganisation and membranous vacuole formation. Interestingly, when epimastigotes and trypomastigotes were treated with different diterpenes, only the epimastigotes were affected by treatment and lost membrane permeability.

Not all of the diterpene acids had similar effects (Fig. 2). This result suggests that different isolated compounds have different mechanisms of action to kill Leishmania cells. Despite the different activity levels of diterpene acids against $L$. amazonensis, the use of diterpene acids in the form of oleoresin or as combinations of compounds obtained from the same plant may yield better results than those obtained from the use of the isolated compounds. In a recent study, Izumi et al. (2012) demonstrated that copalic acid and $\beta$-caryophyllene exhibit synergistic activity against Trypanosoma cruzi. Many species of the genus Copaifera contain both of these compounds and other diterpenes evaluated in our study. We suggest that terpenes present in the copaiba oil can work synergistically to facilitate better parasitic inhibition, which is one of the hypotheses regarding its function in the plant. 

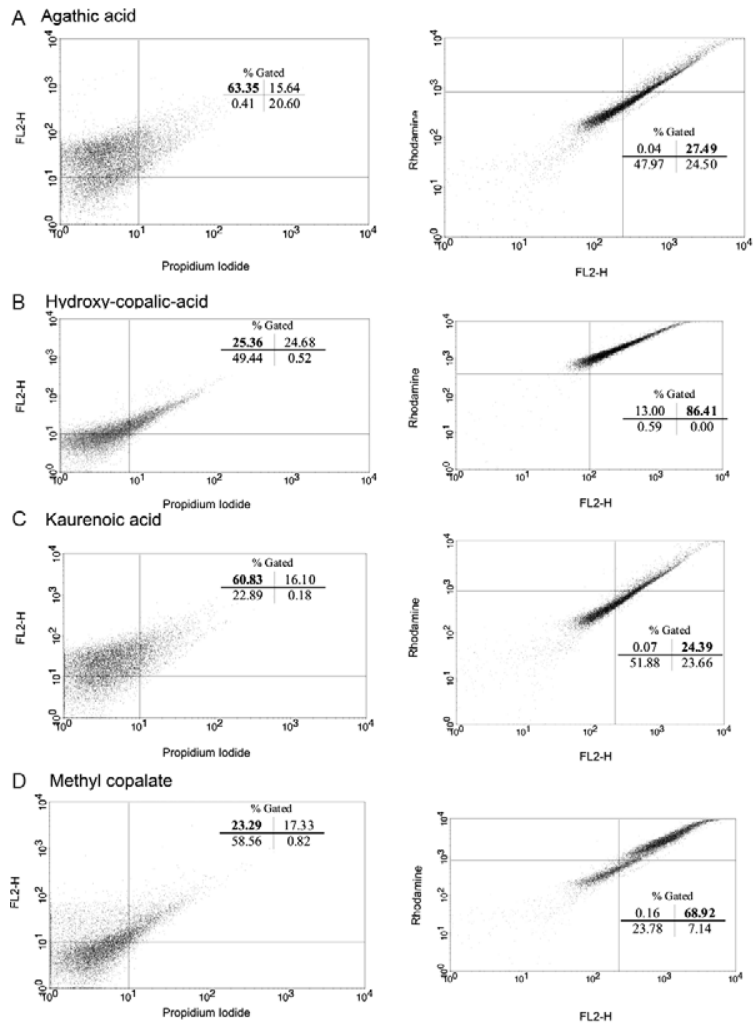
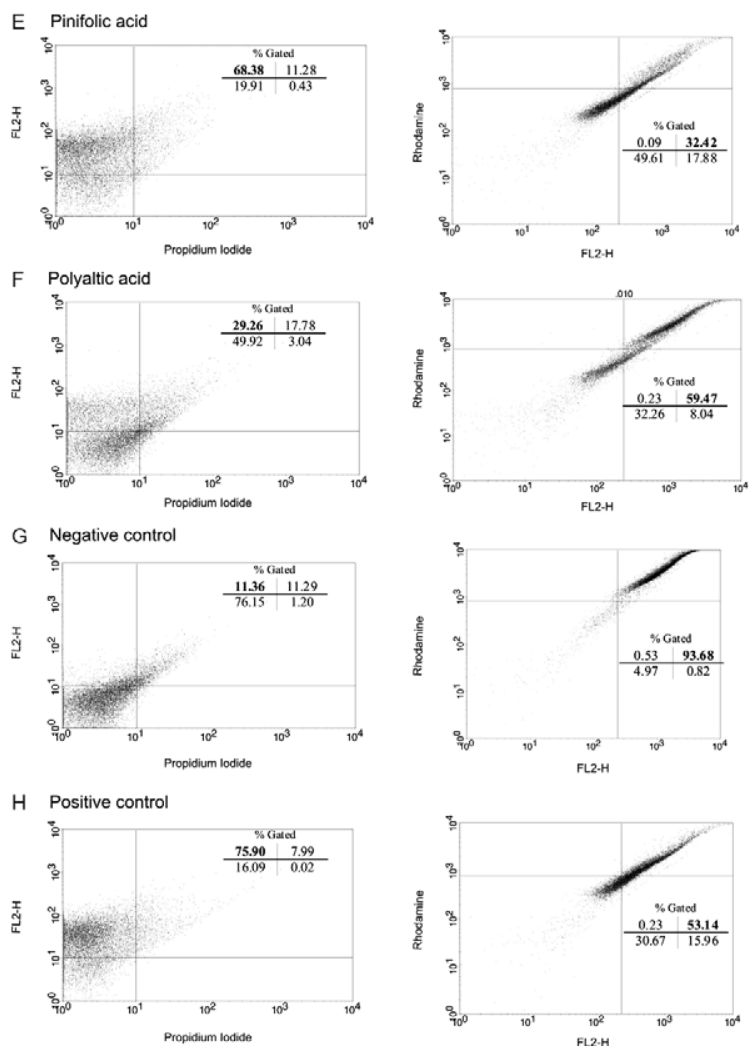

Fig. 2: flow cytometry analysis of axenic amastigotes of Leishmania amazonensis. A: agathic acid; B: hydroxycopalic acid; C: kaurenoic acid; D: methyl copalate; E: pinifolic acid; F: polyaltic acid; G: control cells without treatment; H: positive control; left column: studying cell viability; right column: studying of mitochondrial membrane potential. Treatment with $100 \mu \mathrm{g} / \mathrm{mL}$ for $3 \mathrm{~h}$. Analysis of 10,000 events with the percentage of gated cells indicated in the quadrants. FL2-H: fluorescence.

All of the diterpene acids from copaiba oil that were screened in this study had some level of activity against L. amazonensis. Hydroxycopalic acid and methyl copalate exhibited the most activity against promastigotes. However, pinifolic acid and kaurenoic acid exhibited the most activity against axenic amastigotes. The isolated compounds agathic acid, kaurenoic acid and pinifolic acid yielded significant increases in plasma membrane permeability and mitochondrial membrane depolarisation. In conclusion, copaiba oil and its diterpene acids should be further explored for the development of new antileishmanial drugs.

\section{REFERENCES}

Aï-Oudhia K, Gazanion E, Vergnes B, Oury B, Sereno D 2011. Leishmania antimony resistance: what we know what we can learn from the field. Parasitol Res 109: 1225-1232.

Cruz AK, de Toledo JS, Falade M, Terrão MC, Kamchonwongpaisan S, Kyle DE, Uthaipibull C 2009. Current treatment and drug discovery against Leishmania spp and Plasmodium spp - a review. Curr Drug Targets 10: 178-192.

Delorenzi JC, Attias M, Gattass CR, Andrade M, Rezende C, Pinto AC, Henriques AT, Bou-habib DC, Saraiva BEM 2001. Antileishmanial activity of indoll alkaloid from Peschiera australis. Antimicrob Agents Chemother 45: 1349-1354.

Gelb MH, Hol WG 2002. Drugs to combat tropical protozoan parasites. Science 297: 343-344.
Izumi E, Ueda-Nakamura T, Veiga-Junior VF, Pinto AC, Nakamura CV 2012. Terpenes from Copaifera demonstrated in vitro antiparasitic and synergic activity. J Med Chem 55: 2994-3001.

Kaye P, Scott P 2011. Leishmaniasis: complexity at the host-pathogen interface. Nat Rev Microbiol 9: 604-615.

Murray HW 2010. Treatment of visceral leishmaniasis in direction from Bihar State, India. Future Microbiol 5: 1301-1303.

Murray HW, Berman JD, Davies CR, Saravia NG 2005. Advances in leishmaniasis. Lancet 366: 1561-1577.

Rodrigues JCF, Bernardes CF, Visbal G, Urbina JA, Vercesi AE, Souza W 2007. Sterol methenyl tranferase inhibitors alter the ultrastructure and function of the Leishmania amazonensis mitochondrion leading to potent growth inhibition. Protist 158: 447-456.

Rosa MSS, Mendonça-Filho RR, Bizzo HR, Rodriguez IA, Soares RMA, Souto-Padrón T, Alviano CS, Lopes AHCS 2003. Antileishmanial activity of a linalool-rich essential oil from Croton cajucara. Antimicrob Agents Chemother 47: 1895-1901.

Santa-Rita RM, Henriques-Pons A, Barbosa HS, Castro SL 2004. Effect of the lysophospholipid analogues edelfosine, ilmefosine and miltefosine against Leishmania amazonensis. J Antimicrob Chemother 54: 704-710.

Santos AO, Costa MA, Ueda-Nakamura T, Dias-Filho BP, VeigaJúnior VF, Souza-Lima MM, Nakamura CV 2011. Leishmania amazonensis: effects of oral treatment with copaiba oil in mice. Exp Parasitol 129: 145-151.

Santos AO, Ueda-Nakamura T, Dias-Filho BP, Veiga-Jr VF, Nakamura CV 2012. Copaiba Oil: an alternative to development of new 
drugs against leishmaniasis. Evid Based Complement Alternat Med: 898419.

Santos AO, Ueda-Nakamura T, Dias-Filho BP, Veiga-Junior VF, Pinto AC, Nakamura, CV 2008. Effect of Brazilian copaiba oils on Leishmania amazonensis. J Ethnopharmacol 120: 204-208.

Santos AO, Veiga-Santos P, Ueda-Nakamura T, Dias-Filho BP, Sudatti DB, Bianco E M, Pereira RC, Nakamura CV 2010. Effect of elatol, isolated from red seaweed Laurencia dendroidea, on Leishmania amazonensis. Mar Drugs 8: 2733-2743.

Seifert K 2011. Structures, targets and recent approaches in antiLeishmania drug discovery and development. Open Med Chem J 5: 31-39.
Tiuman TS, Santos AO, Ueda-Nakamura T, Dias-Filho BP, Nakamura CV 2011. Recent advances in leishmaniasis treatment. Int J Infect Dis 15: 525-532.

Ueda-Nakamura T, Attias M, Souza W 2001. Megasome biogenesis in Leishmania amazonensis: a morphometric and cytochemical study. Parasitol Res 87: 89-97.

Ueda-Nakamura T, Mendonça-Filho RR, Morgado-Diaz JA, Maza PK, Dias-Filho BP, Cortez DAG, Alviano DS, Rosa MS, Lopes AHCS, Alviano CS, Nakamura CV 2006. Antileishmanial activity of eugenol-rich essential oil from Ocimum gratissimum. Parasitol Int 55: 99-105.

WHO - World Health Organization 2011. Urbanization: an increasing risk factor for leishmaniasis. Available from: who.int/wer>. 